\title{
Standardsprache im DaF-Unterricht: Normstandard - nationale Standardvarietäten - regionale Standardvarietäten
}

Helmut Spiekermann (Freiburg i. Br.)

\begin{abstract}
In this article I shall address the following question: which kind of the Standard German should be integrated into the course curriculum of "German as a foreign language"? In order to answer this question, however, it is argued that Standard German is by no means homogenous, rather a distinction must be drawn between national and regional standard varieties. The "standard" variety typically taught in these courses neither exists as an ideal standard variety used in everyday speech, nor does it adequately portray the actual linguistic situation. In this article it is therefore advocated that both the variability of the standard as well as the results of the latest sociolinguistic research be taken into account when designing the curriculum for "German as a foreign language".
\end{abstract}

\section{$1 \quad$ Welches Deutsch soll man lehren?}

Die Frage, wie der Standardspracherwerb in der Fremdsprache Deutsch bei Kindern und Jugendlichen mit Migrationshintergrund in einer dialektsprechenden Umgebung verbessert werden kann, ${ }^{1}$ lässt sich aus sehr unterschiedlichen Perspektiven beantworten. Neben der Aufgabe, sprachlichen Förderbedarf zu erkennen und geeignete Methoden zur Vermittlung des Standarddeutschen zu entwickeln, stellt sich meiner Ansicht nach immer noch (cf. Hensel 2000, Krumm 2006 u. a.) die Frage, welches Deutsch gelehrt werden soll. Selbst wenn man einen Konsens dahingehend findet, dass es primär um die Vermittlung der deutschen Standardsprache geht, hat man mit dieser Aussage noch keine eindeutige Auswahl der zu vermittelnden Sprachform getroffen. Die deutsche Standardsprache weist sowohl in ihrer geschriebenen als auch in ihrer gesprochenen Form regionale Varianten auf, auf die in der Vermittlung der Standardsprache im Unterricht "Deutsch als Fremdsprache" eingegangen werden sollte. Die Möglichkeit zur Berücksichtigung unterschiedlicher Standardvarietäten ist in den vergangenen Jahren durch einige neue empirische Arbeiten zumindest für einen Teil des deutschen Sprachgebietes geschaffen worden. Im vorliegenden Beitrag soll in diesem Zusammenhang auf einige theoretische und empirische Argumente hingewiesen werden, anhand derer Variation am Standardpol beschrieben und für den Deutschunterricht nutzbar gemacht werden kann.

\section{$2 \quad$ Variabilität des Deutschen}

Die deutsche Sprache zeichnet sich dadurch aus, dass sie in eine Vielzahl unterschiedlicher Varietäten aufgesplittert ist. Diese Varietäten werden i.d. R. durch außersprachlichen Beschreibungsdimensionen kategorisiert, von denen die folgenden in der Forschungsliteratur

\footnotetext{
${ }^{1}$ Dies ist die zentrale Leitfrage des Projekts "Deutsch als Zweitsprache in Dialektumgebung" in Bern/CH (im Internet unter http: //www.karmancenter.unibe.ch/zweitsprache/index_html.de), dem ich den Anstoß für diesen Aufsatz verdanke.
} 
(cf. Dittmar 1997) häufig für eine erste grobe Gliederung herangezogen werden: Zeit (diachrone Variation), soziale Schicht/Gruppe (diastratische Variation), Situation (diaphasische Variation) und Raum (diatopische Variation). Ich werde mich im Folgenden auf die diatopische Variation konzentrieren, da diese für die standardsprachlicher Variation entscheidend ist. ${ }^{2}$

\subsection{Modellierung regionaler Variation}

Am Anfang soll ein Versuch der Modellierung regionaler Variation im Deutschen stehen, wie man sie für einen großen Teil des deutschen Sprachgebiets annehmen kann, in dem zwischen den Extrempolen Dialekt und Standardsprache entlang eines sprachlichen Kontinuums regionale Zwischenformen anzusetzen sind (Diaglossie). Das Modell ist gültig für Süddeutschland, weite Teile des Mitteldeutschen und für Österreich. ${ }^{3}$

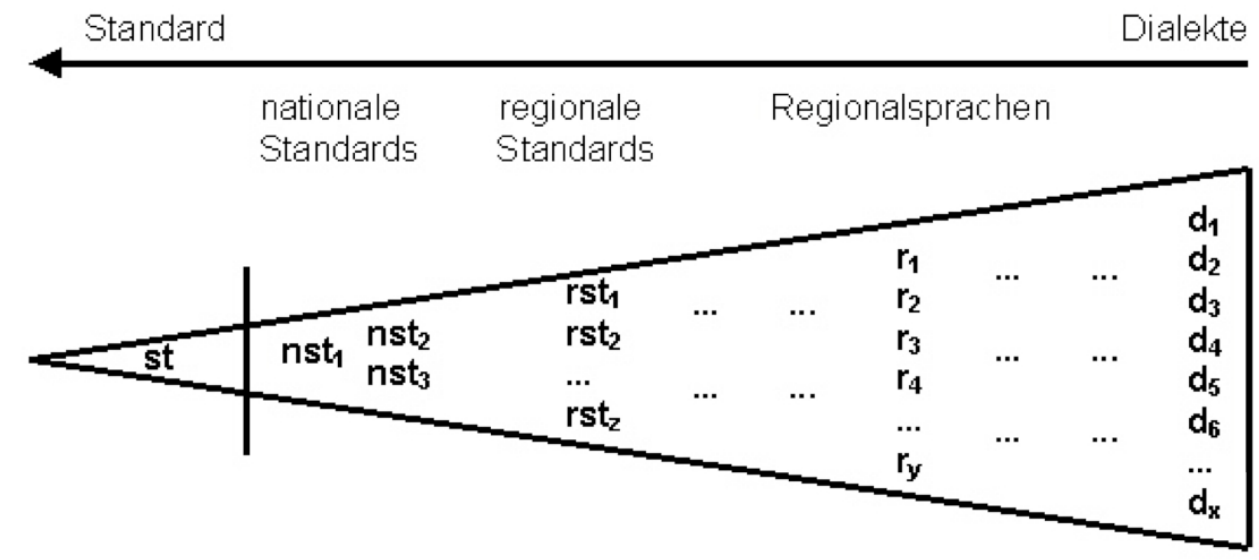

Abb. 1: Modell regionaler Varietäten - Diaglossie, nach Baßler/Spiekermann (2001)

Die Fahnenform des Modells (Abb. 1) soll andeuten, dass die verschiedenen Varietäten unterschiedlich stark ausdifferenziert sind, d.h. dass unterschiedlich viele Varietätenrealisierungen anzunehmen sind. Wir können davon ausgehen, dass es - bezogen auf das Deutsche - eine Vielzahl unterschiedlicher Dialekte gibt. Unterschiedliche Regionalsprachen, die häufig als Ausgleichssprachen zwischen Dialekten und als Anpassung an einen Standard entstanden sind, kommen in geringerer Zahl vor, Standardvarietäten noch seltener. Die Fahne spitzt sich zum Standardpol hin also zu. Die Punkte links und rechts der Symbole für Regionalsprachen sollen andeuten, dass zu den Dialekten und zu den Standardvarietäten ein fließender Übergang anzunehmen ist, d. h. ein Kontinuum von Varietäten, die sich z. T. nur minimal voneinander unterscheiden. Als Einteilungskriterien werden häufig folgende außersprachliche Faktoren diskutiert:

a. Die kommunikative Reichweite: Wie stark ist die Varietät in ihrer Verbreitung eingeschränkt, oder anders gesagt: Wie groß ist der Radius um den Gebrauchsort herum, in dem man sich noch in der Varietät mit anderen verständigen kann? Die kommunikative Reichweite ist bei der Standardsprache am größten, da sie grundsätzlich im gesamten deutschsprachigen Gebiet als Kommunikationsform eingesetzt werden kann. Sie ist bei Dialekten am geringsten und im Extremfall auf die Kommunikations-

\footnotetext{
2 Ausführliche Beschreibungen der Variation des Deutschen nach unterschiedlichen Beschreibungsdimensionen finden sich u. a. bei Dittmar (1997), Barbour/Stevenson (1998) und Löffler (1994).

${ }^{3}$ Zu Modellierungen der Standard-Dialekt-Verhältnisse cf. auch Schmidt (1998), Ammon (2003), Auer (2005).
} 

nationale Standardvarietäten - regionale Standardvarietäten

gemeinschaft eines Ortes beschränkt. Die Regionalsprache nimmt eine Zwischenstellung ein.

b. Die Verwendung dialektaler Merkmale: Dieses Kriterium hängt eng mit dem ersten zusammen. Gemeint ist damit, dass unterschiedlich viele dialektale, kleinräumig verbreitete Merkmale in den Varietäten vorkommen. Die Standardsprache ist als die Varietät definiert, die grundsätzlich keine bzw. nur sehr wenige Dialektmerkmale enthält. Wir werden an späterer Stelle sehen, dass dies für die nationalen und regionalen Standardvarietäten in geringerem Maße zutrifft. In den Dialekten finden wir - natürlich - die meisten Dialektmerkmale. Die Regionalsprachen nehmen eine Zwischenstellung ein.

c. Die Gesprächssituation: Soziolinguistische Untersuchungen haben gezeigt, dass die Varietäten sich deutlich in ihrem situationsspezifischen Gebrauch unterscheiden. Man kann sagen, dass die Domäne der Standardsprache die formelle Situation ist. In Vorträgen, in Fernsehdiskussionen und bei anderen Gelegenheiten, bei denen ein Sprecher/eine Sprecherin sich an eine größere Gruppe von Zuhörern richtet, wird die Varietät verwendet, von der man sich die größtmöglichste Verständlichkeit verspricht. In informellen, privaten Situationen ist das häufig anders. In der Familie oder unter Freunden gebraucht man sehr viel häufiger den Dialekt. ${ }^{4}$ In den deutschsprachigen Regionen, in denen der Dialekt stark zurückgedrängt ist (vor allem in Norddeutschland), wird allerdings oft eine standardnahe Varietät auch in informellen Situationen verwendet.

d. Die sprachliche Norm: Die Standardsprache zeichnet sich dadurch aus, dass sie genormt in Form von so genannten Kodizes vorliegt. Man kann z. B. im Duden nachschlagen, wie ein Wort der Standardsprache geschrieben, ausgesprochen oder in einen grammatischen Kontext eingefügt werden kann bzw. muss. Die nationalen Standards definieren sich über die nationalen Kodizes Deutschlands, Österreichs und der Schweiz. Da in diesen drei großen deutschsprachigen Ländern unterschiedliche Kodizes gebräuchlich sind (cf. Ammon 1995), lassen sich drei nationale Standardvarietäten differenzieren. Zum Dialektpol hin nimmt die Normtreue - immer bezogen auf die standardsprachliche Norm - immer mehr ab. Die Dialekte folgen häufig eigenen (nicht genormten und nicht kodifizierten!) Regeln und sind so gesehen am weitesten von der Standardnorm entfernt.

Die Regionalsprache (auch: Umgangssprache) ${ }^{5}$ wird in nicht zu formellen, aber auch nicht zu informellen Situationen, also z. B. im Gespräch mit Fremden verwendet, sie zeigt eine gewisse Orientierung an der standardsprachlichen Norm, ohne diese aber jemals zu erreichen, sie weist eine Vielzahl dialektaler Merkmale auf, ist aber dennoch relativ weiträumig verständlich.

Für die Schweiz und für weite Teile Norddeutschlands muss das Modell angepasst werden, da hier - in Norddeutschland gilt dies zumindest für die Teile, in denen der niederdeutsche Dialekt noch lebendig ist - zwischen der Standardsprache und den Dialekten keine Übergangsformen anzusetzen sind. Die "Fahne" im Modell ist sozusagen in der Mitte in zwei nicht miteinander verbundene Teile zerschnitten (cf. Abb. 2). Dialekt und Standard stehen in einem diglossischen Verhältnis zueinander. In der Schweiz (anders als in Norddeutschland) ist der Gebrauch des Standards größtenteils auf die Schriftsprache beschränkt (mediale Diglossie).

\footnotetext{
${ }^{4}$ Cf. hierzu z. B. die Ergebnisse aus Umfragen zur (subjektiv wahrgenommenen) Dialektkomptenz in Niebaum/Macha (2006: 165-171).

5 Zum Problem des Begriffs "Umgangssprache" cf. schon Bichel (1973).
} 


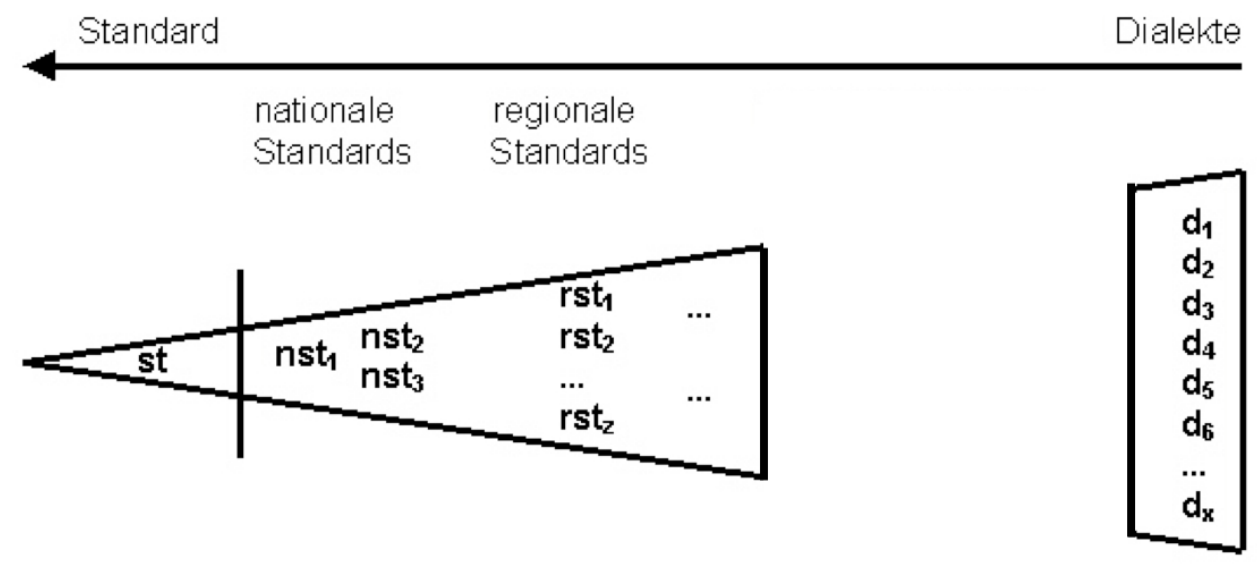

Abb. 2: Modell regionaler Varietäten - Diglossie, nach Baßler/Spiekermann (2001)

Nicht wenige Dialektologen und Soziolinguisten gehen heute davon aus, dass die traditionellen diaglossischen und diglossischen Systeme durch zwei in den vergangenen 50 Jahren stärker werdende Prozesse erheblich verändert werden. Bei diesen Prozessen handelt es sich einerseits um einen Abbau dialektaler Formen (Dialektabbau) und andererseits um eine zunehmende Abkehr von standardsprachlichen Normen (Standardabbau). Ergebnis dieser Entwicklungen war die Ausbildung der neuen Substandardvarietäten (Bellmann 1983), zu denen u. a. die regionalen Standardvarietäten gezählt werden können. Ursachen für diese Entwicklung sind einerseits der zunehmende Gebrauch überregional verständlicher Formen als Folge u. a. größerer Mobilität in der Gesellschaft, der zum Rückgang des Dialektgebrauchs führt, und andererseits eine besonders seit Mitte des 20. Jahrhunderts einsetzende zunehmende Normskepsis, die den Gebrauch nicht-standardsprachlicher Formen fördert und sich insbesondere auf den Gebrauch nationaler Standardvarietäten (s. u.) auswirkt (Standardabbau). Variation nimmt aufgrund dieser Entwicklungen diachron am Dialektpol ab, am Standardpol dagegen zu, da insbesondere regionale Standardvarietäten sich erst seit Mitte des 20. Jahrhunderts zu etablieren beginnen (cf. Mattheier 2003). In den Modellen für die Diaglossie und die Diglossie lassen sich die Entwicklungen wie folgt berücksichtigen (der Abbau wird durch transparente Felder angedeutet):

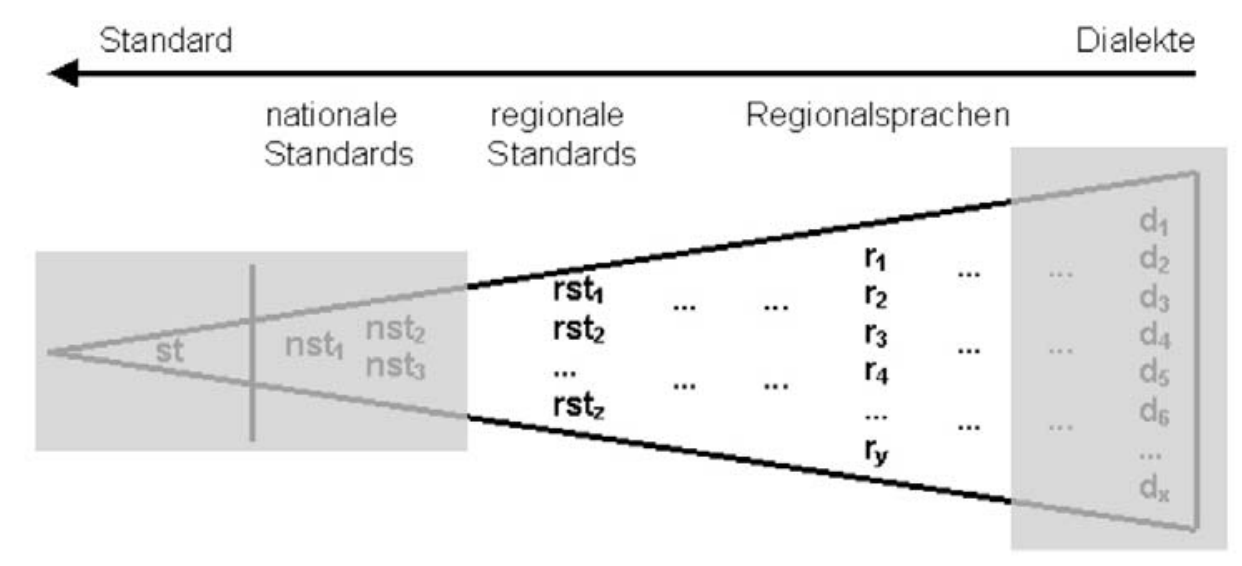

Abb. 3: Modell regionaler Varietäten - Diaglossie, nach Baßler/Spiekermann (2001)

In der Diaglossie wird das Varietätenspektrum so "zusammengedrückt, dass praktisch nur noch die Varietäten im Zentrum erhalten bleiben. Dramatischer wirkt sich besonders der 
Dialektabbau in der Diglossie aus, so wie sie vor allem in Norddeutschland begegnet: 6 Sprecher verfügen kaum über unterschiedliche regionale Varietäten, sondern i. d. R. nur über einen regionalen Standard und diesem sehr ähnliche Regionalsprachen, die die kommunikativen Funktionen des Dialekts übernehmen können und zu Nähesprachen werden (in Abb. 4 die Punkte neben den Symbolen für den Regionalstandard). Die regionale Variation ist hier auf dem Weg zur Monoglossie.

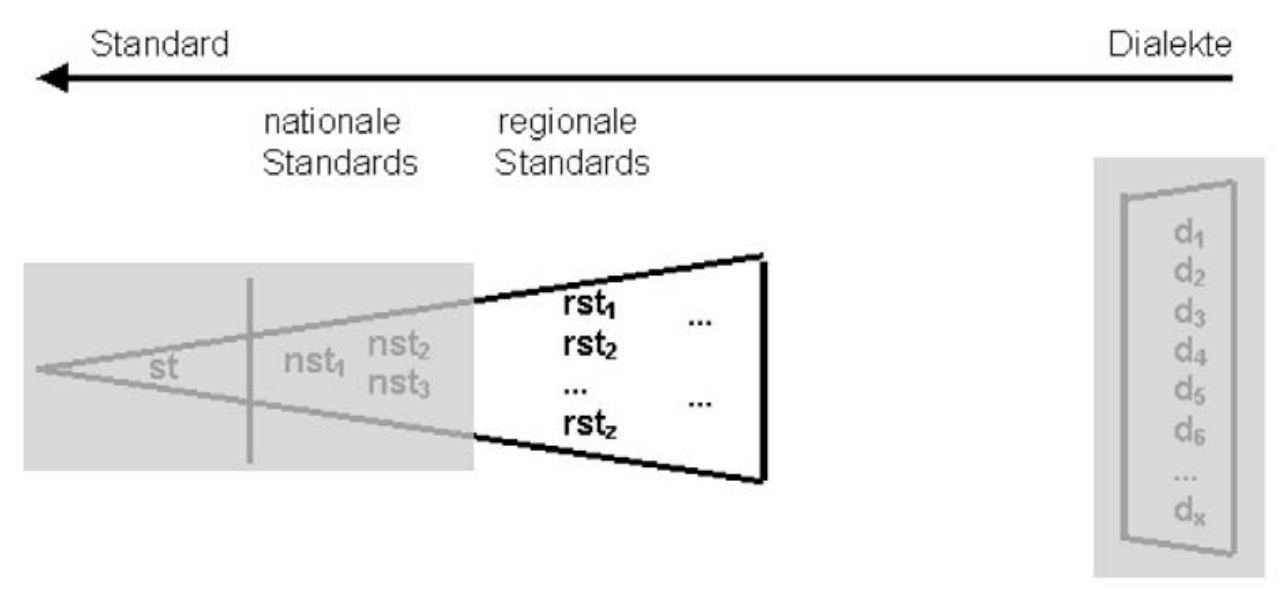

Abb. 4: Modell regionaler Varietäten - Diglossie, nach Baßler/Spiekermann (2001)

Die Entwicklungen der letzten Jahre und Jahrzehnte wirken sich also u. a. erheblich auf die standardsprachlichen Varietäten aus. Insbesondere regionale Standardvarietäten gewinnen nach diesen Modellen an Bedeutung.

\subsection{Normstandard - nationale Standardvarietäten}

Nachdem Standardvarietäten in ihrem Verhältnis zu anderen regionalen Varietäten im Modell beschrieben sind, stellt sich als nächstes die Frage, wie sie konkret aussehen.

Immer wieder, wenn von der deutschen Standardsprache die Rede ist, dominiert das Bild von dem einem, dem einheitlichen Standard die Diskussion. Geht man von der in den Wörterbüchern, Aussprachewörterbüchern und Grammatiken kodifizierten Sprachform aus und nennt diese "Standard", dann muss man sie als theoretisches Konstrukt bezeichnen. Tatsächlich wird es niemanden geben, der diesen Standard perfekt beherrscht. Selbst geschulte Sprecher wie Schauspieler oder Nachrichtensprecher sind nicht in der Lage, einen völlig variationsfreien oder von regionalen Einflüssen unberührten Standard zu artikulieren. Aus diesem Grund hat sich die Soziolinguistik und mit ihr die moderne Dialektologie längst von der Vorstellung distanziert, dass es den Standard, die eine Standardsprache gibt. An deren Stelle sind Gruppen von Standardvarietäten getreten, die häufig als nationale und regionale Standardvarietäten unterschieden werden.

Die deutsche Standardsprache wird in ihrer Einheitlichkeit durch die Tatsache eingeschränkt, dass sie in unterschiedlichen Nationen Amtssprache ist. Normierung und Kodifizierung einer Standardsprache/Amtssprache liegt in der Entscheidungsgewalt souveräner Nationen, so dass es nicht verwundern muss, dass die deutsche Standardsprache in verschiedene nationale Standardvarietäten zerfällt. Deutsch ist nationale Amtssprache in der Schweiz, Österreich, Liechtenstein, Luxemburg und Deutschland. In Südtirol und Ostbelgien ist es außerdem regionale Amtssprache. Die drei großen deutschsprachigen Nationen verfügen über eigene Sprachkodizes, d. h. eigene Wörterbücher, Orthographien und Aussprachelexika, so dass

\footnotetext{
${ }^{6}$ In der Schweiz ist der Dialektabbau deutlich schwächer ausgeprägt bzw. nicht zu beobachten.
} 
heute drei nationale Standardvarietäten unterschieden werden können. Die Unterscheidung in eine schweizerische, eine österreichische und eine deutsche Standardvarietät folgt dem Konzept der Plurizentrizität des Deutschen (Clyne 1992, Ammon 1995). Lexeme, die nur in einem der drei großen Nationen verwendet werden, werden von Ammon als Helvetismen, Austriazismen und Teutonismen unterschieden. Ammon definiert nationale Varietäten durch sog. nationale Varianten, d. h. Lexemvarianten, die nur in einer der deutschsprachigen Nationen gebräuchlich sind. Folgender Ausschnitt aus einem Zeitungsartikel einer überregional erscheinenden schweizerischen Tageszeitung gibt ein Beispiel für nationale Standardvarietäten in ihrer geschriebenen Form. ${ }^{7}$

\section{Kantersieg im Stadtderby - GC-Gegner Porto für Rückspiel gerüstet}

geb. Porto, 20 August

Zum dritten Mal innert drei Monaten hat Porto den Titelhalter Boavista im Derby der "Unbesiegten Stadt" geschlagen. Einem bedeutungslosen 4: 0 zum Abschluss der vergangenen Saison (Hattrick Deco) und dem 1: 0 im Supercup vor vierzehn Tagen liessen die Portistas am Sonntagabend im zweiten Meisterschaftsmatch vor 30.000 Besuchern im Estadio das Antas bei Regen und herbstlichen Temperaturen ein deutliches 4: 1 folgen. Der Match war allerdings bis in die Schlussphase hart umkämpft. Nach mehreren Abprallern und Konfusionen glückte Teamsenior Jorge Costa in der 79. Minute das erlösende Tor zum 2: 1. Als Pedro Emanuel kurz darauf des Feldes verwiesen wurde, nutzten der beste Spieler auf dem Platz, Jorge Andrade, und "Joker" Helder Postiga die Desorientierung des Gegners zur Resultaterhöhung.

(aus: Neue Zürcher Zeitung; 21.08.2001, 47)

Zeitungssprache wie die in obigem Beispiel ist i.d. R. (aber nicht immer!) als Standardsprache anzusehen. Durch die beabsichtigte überregionale Verbreitung kann man davon ausgehen, dass die Verfasser auf eine Varietät zurückgreifen, die eben diese ermöglicht, also auf die Standardsprache. Eine einfache Analyse des Textes (mit einem bundesdeutschen Standard als Vergleichsbasis) lässt Besonderheiten des schweizerischen Standards in folgenden linguistischen Teilbereichen erkennen:

In der Orthographie

1. <liessen>: Der schweizerdeutsche Standard setzt grundsätzlich immer <ss> anstelle von $<\beta>$. Die jüngste Rechtschreibreform hat in den deutschsprachigen Nationen außerhalb der Schweiz lediglich $<\beta>$ nach Kurzvokale durch $<$ ss $>$ ersetzt. Nach Langvokalen blieb $<\beta>$ erhalten.

In der Morphologie

1. <der Match>: Genusschwankung, denn bundesdeutsch ist es $<$ das Match $>$.

2. $<$ Resultaterhöhung $>$ : Bundesdeutsch wäre hier ein sog. Fugen- $<$ s $>$ nötig (also: $<$ Resultat+s+erhöhung $>$ ).

In der Lexik

1. <innert>: Bundesdeutsch würde man hier <innerhalb $>$ verwenden.

2. $<$ Titelhalter $>$ : Bundesdeutsch $<$ Titelträger $>$.

3. $<$ Meisterschaftsmatch $>$ : Anstelle von $<$ Match $>$ (engl. Einfluss - im schweizerischen Standard sind grundsätzlich häufiger Fremdwörter zu finden als im bundesdeutschen) steht im bundesdeutschen Standard i. d. R. $<$ Spiel $>$.

4. <Teamsenior>: Im bundesdeutschen Standard würde man vielleicht <dem ältesten Spieler> oder dgl. sagen.

\footnotetext{
${ }^{7}$ Ich beschränke mich hier auf einen Vergleich des schweizerischen und bundesdeutschen Standards. Für den österreichischen Standard können entsprechende Analysen vorgenommen werden.
} 
Wie die Analyse zeigt, stehen Unterschiede in der Lexik in schriftlichen Standardformen der Schweiz und Deutschlands im Vordergrund. Unterschiede in diesem Bereich sind aber durchaus nicht die einzigen, die die beiden nationalen Standardvarietäten voneinander unterscheiden. Ammon (1995) nennt darüber hinaus in den gesprochenen Standardvarietäten phonologische Unterschiede, zu denen (wiederum aus Sicht des bundesdeutschen Standards) in der schweizerischen Varietät z. B. die deutlich gerollte Artikulation von $/ \mathrm{r} /$ auch in postvokalischer Position (also z. B. in ['dort], <dort> (im bundesdeutschen Standard wird das /r/ in dieser Position i. d. R. vokalisiert, also z. B. ['dopt]), die Affrizierung von /k/ (z. B. [kxom], <komm>) oder die häufige velare Artikulation des Ich-Lauts (z. B. [milx], <Milch>) gehören (cf. auch Flüe-Fleck/Hove 1994, Hove 1995, Christen 2001). Nationale Varianten als Kennzeichen nationaler Standardvarietäten begegnen also in unterschiedlichster Form sowohl in geschriebener als auch in gesprochener Sprache.

\subsection{Regionale Standardvarietäten}

Neben den Konzept der Plurizentrizität (auch: Plurinationalität), das bei der Unterscheidung nationaler Standardvarietäten angewendet werden kann, ist das der Pluriarealität (Ammon 1998) für die Variabilität der Standardsprache innerhalb von Nationen verantwortlich zu machen. Variation in der Standardsprache innerhalb Deutschlands macht sich dadurch bemerkbar, dass Sprecher und Sprecherinnen in intendierter Standardsprache bzgl. ihrer regionaler Herkunft einordbar sind. Dies zeigt sich wie im Fall der nationalen Standardvarietäten auf unterschiedlichsten sprachlichen Ebenen. Anders als bei nationalen Standardvarietäten sind regionale allerdings nicht an nationale, politische Grenzen gebunden, sondern können innerhalb dieser kleinräumigere Einheiten bilden oder aber diese überschreiten, besonders dann, wenn Dialekträume sich über mehrere Staaten erstrecken, wie dies für das Bairische oder Alemannische der Fall ist. ${ }^{8}$ Besonders gut untersucht ist die regionale Variation im Bereich des Wortschatzes. Die Untersuchungen von Eichhoff (1977) und Elspaß/Möller (2006) beziehen sich auf die Umgangssprache bzw. Alltagssprache, sie lassen sich aber zweifellos als Spiegel des Standardsprachgebrauchs ansehen, da Umgangsund Alltagssprache in Teilen des deutschen Sprachgebiets mit standardnahen Varietäten gleichzusetzen sind (besonders im Norden) und in anderen Teilen zu diesen aufgrund des durch die Diaglossie bestehenden sprachlichen Kontinuums in einem engen Verhältnis stehen (besonders im Süden). Dies bestätigen auch zahlreiche Einzelergebnisse der Untersuchung von Ammon et al. (2004) zum Standard in Deutschland, Österreich und der Schweiz, die in Form eines Variantenwörterbuchs der deutschen Sprache veröffentlicht sind. Eichinger (2001) argumentiert, dass viele Unterschiede in der Standardsprache sich als Gegensatz zwischen einer nördlichen und einer südlichen Variante darstellen, so auch im Wortschatz. Viele Beispiele aus Eichhoff belegen das. So ist das Lexem <Junge> in zwei Hauptvarianten nämlich <Junge $>$ im Norden und $<$ Bub $>$ im Süden - geteilt (cf. Abb. 5).

\footnotetext{
${ }^{8}$ Dass Staatsgrenzen hier aber durchaus abgrenzende Funktionen haben und historisch eng verbundene Dialekte trennen können, hat zuletzt Auer (2004) gezeigt. Von daher zeigt sich hier, dass nationale und regionale Standardvarietäten einander in ihrer Gültigkeit beeinflussen.
} 


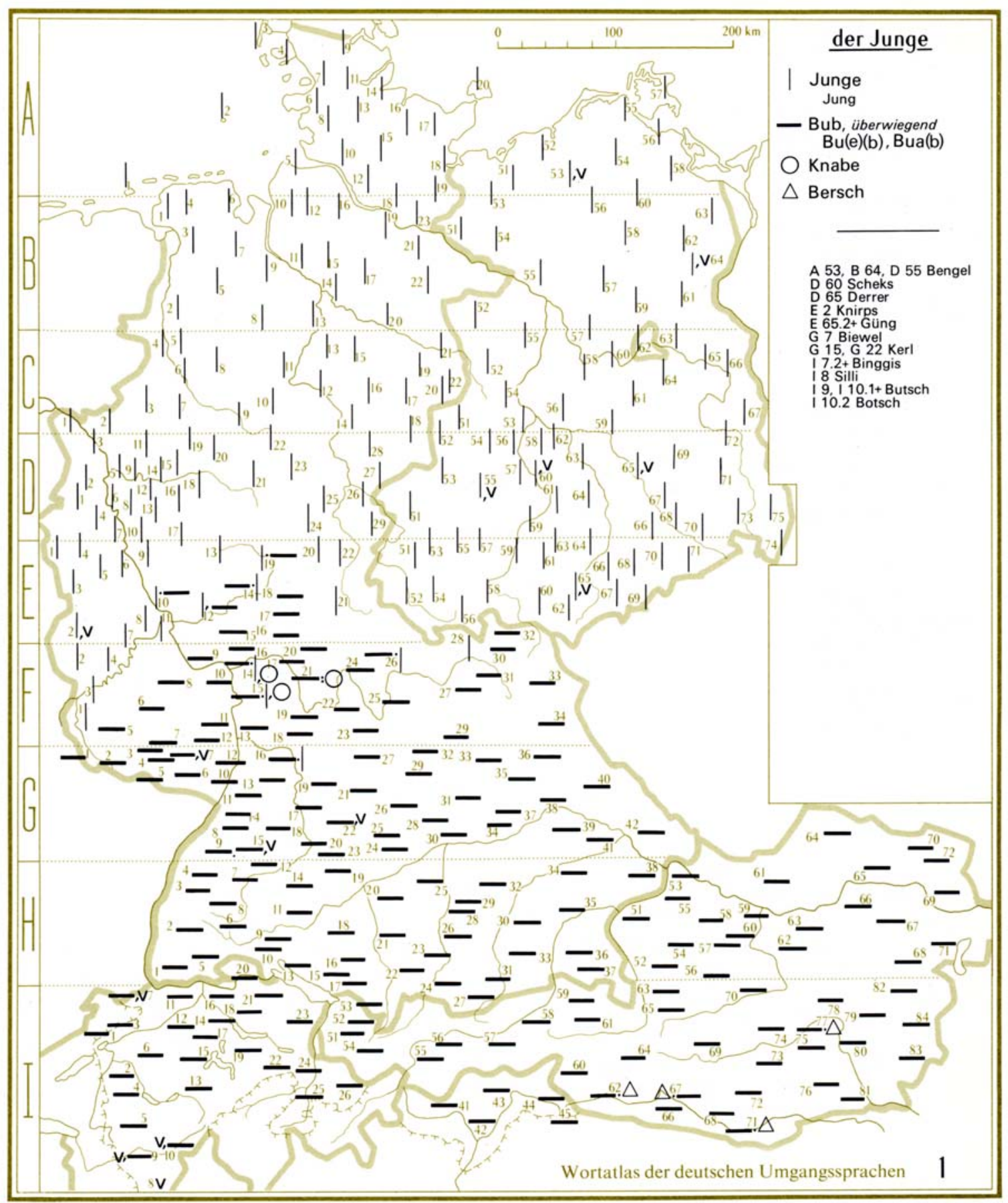

Abb. 5: Umgangssprachliche Variation im Lexem <Junge>, Eichhoff (1977: Karte 1)

Die Frage, wie viele regionale Standardvarietäten es im deutschen Sprachraum gibt, lässt sich dennoch sicherlich nicht mit "zwei" beantworten. Die Anzahl der regionalen Standards liegt vermutlich deutlich höher.

Berend (2005) differenziert nach den Ergebnissen ihrer Untersuchung zu Gebrauchsstandards in den alten Bundesländern vier Sprechstandards. Untersuchungsgegenstand waren bei ihr Spontandaten (standardsprachliche Interviews) aus den 1970er Jahren (König-Korpus). Der Gebrauch der Standardsprache wird durch die formelle Situation, in der die Interviews geführt wurden, provoziert. Berend stellt erste Ergebnisse ihrer Untersuchung in Form einer Karte zusammen (cf. Abb. 6), aus der vier gesprochensprachlichen Standardvarietäten mit norddeutscher, mitteldeutscher, südwestdeutscher und südostdeutscher Prägung hervorgehen. 


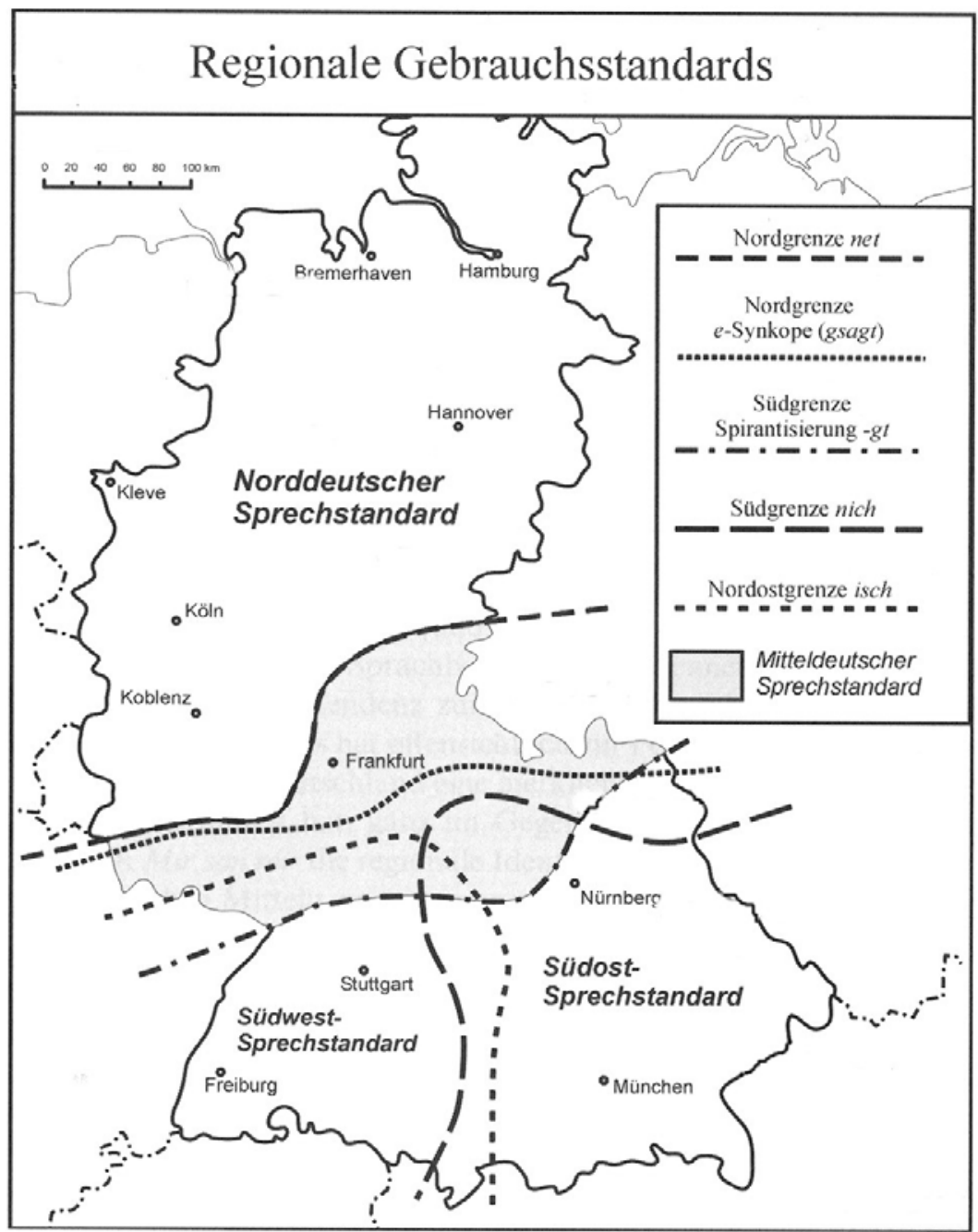

Abb. 6: Regionale Gebrauchsstandards in den alten Bundesländern (Berend 2005: 166)

Die Möglichkeit zur Differenzierung genau dieser vier Sprechstandards ist sicherlich auch durch die von Berend gewählten Merkmalen bedingt, grundsätzlich zeigt ihre Analysemethode aber einen Weg auf, wie empirisch regionale Standardvarietäten ermittelt und voneinander abgegrenzt werden können. Zur Zeit existieren jedoch noch keine Arbeiten, die für das gesamte deutsche Sprachgebiet zuverlässige Aussagen über regionale Standardvarietäten möglich machten. ${ }^{9}$

Dass regionale Standardvarietäten auch kleinräumig untersucht werden können, lässt sich beispielhaft an einer Arbeit demonstrieren, die ich selbst im Südwesten Deutschlands durchgeführt habe. Dort habe ich in den vergangenen Jahren die regionale Standardsprache ausführlich untersucht und einige interessante Ergebnisse erzielen können. Bei den Daten handelt es sich um Interviews in sehr formellen Situationen, so dass wie bei den von Berend untersuchten Daten von dem Gebrauch intendierter Standardsprache ausgegangen werden kann. Es lässt sich feststellen, dass die regionale Markierung standardsprachlicher Sprechweisen zumindest bei älteren Sprechern relativ stark ist. In meiner Untersuchung habe ich die Daten des Pfeffer-Korpus (IDS Mannhein, aufgezeichnet im Jahr 1961) mit neuen Daten verglichen, die ich selbst in den Jahren 2001-2003 in elf Städten in Baden-

\footnotetext{
${ }^{9}$ Das IDS Mannheim versucht dieses Forschungsdesiderat gerade zu beseitigen: In dem Projekt "Variation des gesprochenen Deutsch: Standardsprache - Alltagsprache" unter der Leitung von Nina Berend werden zur Zeit Sprachdaten erhoben, die zur Ermittlung standardsprachlicher Variation auf nationaler und regionaler Ebene herangezogen werden sollen (im Internet unter: http: //www.ids-mannheim.de/prag/AusVar/Deutsch_heute/).
} 
Württemberg erhoben habe (Südwest-Standard-Korpus). Mit Bezug auf die dialektalen Merkmale, die in regionalen Standardvarietäten auftauchen und letztlich die regionale Zuordnung der Sprecher möglich machen, kann man feststellen, dass diese grundsätzlich in der diachronen Perspektive im Rückgang begriffen sind. Man könnte sagen, dass regionale Standardvarietäten grundsätzlich immer weniger regional markiert sind. Dies möchte ich kurz an einigen Beispielen zeigen.

Tabelle 1 gibt einen Überblick über einige der von mir untersuchten Dialektmerkmale. Diese teilen sich in zwei Gruppen: Bezogen auf mein Untersuchungsgebiet - Baden-Württemberg gibt es Merkmale, die nur in Teilen des Gebiets verbreitet sind (Öffnung von /e:/, Hebung von /au/ als schwäbische, Spirantisierung von /g/ und Koronalisierung von /ç/ als fränkische Merkmale), und andere, die in ganz Baden-Württemberg in den zugrunde liegenden Dialekten vorkommen (alle anderen).

\begin{tabular}{|l|l|l|}
\hline Nr. & Merkmal & Beispiel \\
\hline 1 & Öffnung von /e:/ & {$[$ ['lebən], <leben $>$} \\
\hline 2 & Hebung von /au/ & {$[$ hous], <Haus $>10$} \\
\hline 3 & Spirantisierung von /g/ & {$[\mathrm{max}],<$ (ich) mag $>$} \\
\hline 4 & Koronalisierung von /ç/ & $[\mathrm{m} 1]],<\mathrm{mich}>$ \\
\hline 5 & Palatalisierung von /s/ & {$\left[\mathrm{ha} \int\right],<(\mathrm{du})$ hast $>$} \\
\hline 6 & einmorige Realisierung von Vokal+/r/-Verbindung & {$[\mathrm{dot}],<\mathrm{dort}>$} \\
\hline 7 & Lenisierung intervokalischer Fortis & {$[\mathrm{mude}],<$ Mutter $>$} \\
\hline 8 & Gebrauch von $<$ des $>$ anstelle von $<$ das $>$ & \\
\hline
\end{tabular}

Tabelle 1: Übersicht primäre Substandardmerkmale

Die beiden Gruppen von Merkmalen zeigen eine grundsätzlich unterschiedliche Häufigkeit in den von mir untersuchten Daten und sind außerdem in Bezug auf ihre diachrone Entwicklung nicht gleich zu behandeln. Dies wird aus folgendem Diagramm deutlich (cf. auch Spiekermann 2006a, Spiekermann 2006b):

${ }^{10}$ Auch als [h us]. Die Hebung ist im Schwäbischen auf zugrunde liegendes mhd. /û/ beschränkt. 


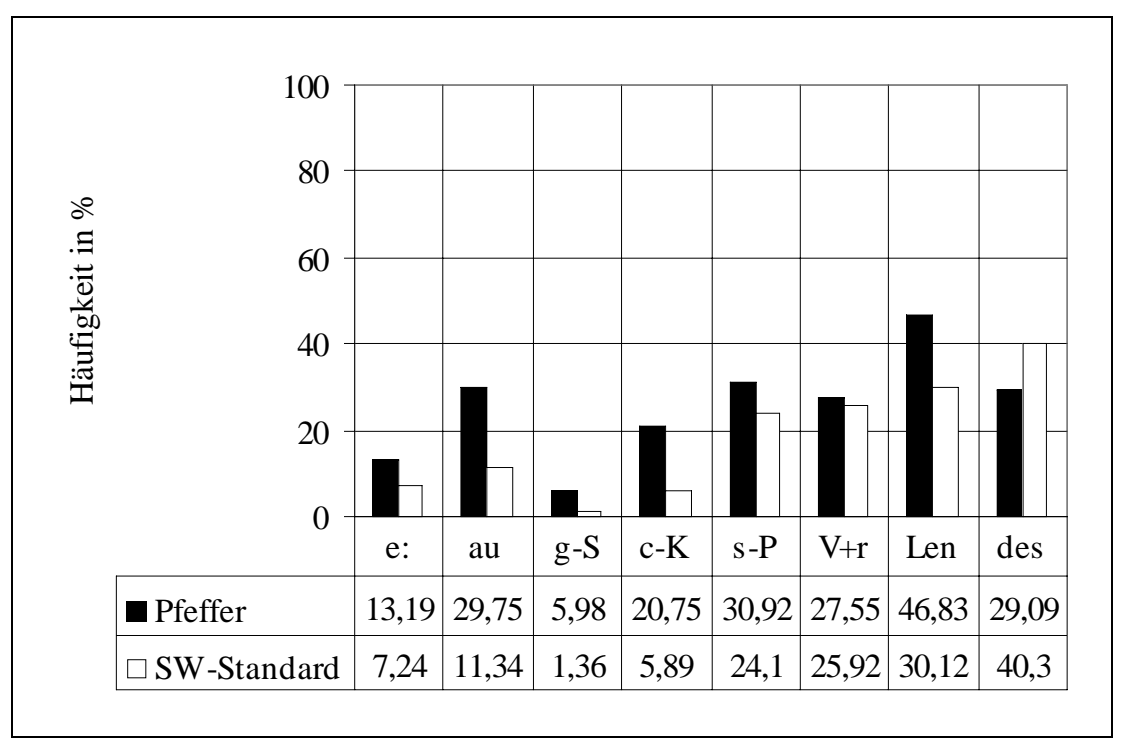

Diagramm 1: Realisierungshäufigkeiten primäre Substandardmerkmale

Dargestellt sind die Ergebnisse für das Südwest-Standard-Korpus (weiße Säulen) und das Pfeffer-Korpus aus dem Jahr 1961 (schwarze Säulen). Die ersten vier Merkmale, allesamt kleinräumig verbreitete Dialektmerkmale, gehen sehr deutlich im Verlauf von 40 Jahren in ihrer Häufigkeit zurück, d. h. der Anteil ihrer Realisierungen geht auf gut 1/3, bei der Öffnung von /e:/ auf etwas mehr als die Hälfte zurück. Die großräumig verbreiteten Merkmale zeigen (mit Ausnahme von <des>) ebenfalls einen Rückgang, jedoch fällt dieser deutlich schwächer aus als bei den kleinräumig verbreiteten Merkmalen. Man kann diesen Befund so zusammenfassen, dass regionale Markierung heute in der Standardsprache nicht mehr über kleinräumig dialektale (also z. B. schwäbische, fränkische etc.) Merkmale stattfindet, sondern über großräumige, d. h. gesamtalemannische bzw. oberdeutsche. Es findet auf der Ebene der regionalen Standardsprachen ein Ausgleich statt, eine Art Standardisierung, die zum Rückgang der kleinräumigen Dialektmerkmale führt.

Neben der Standardisierungstendenz, die im regionalen Standard mit Bezug auf die Dialektmerkmale zu beobachten sind, gibt es eine Destandardisierungstendenz, die sich auf der Ebene der Explizitheit der Lautung zeigt. Von der standardsprachlichen Norm wird in diesen Fällen abgewichen, indem Explizitlautungen verkürzt, verschliffen, vereinfacht werden. Albrecht (2005) nennt diese Merkmale sekundäre Substandmerkmale, im Kontrast zu den dialektalen primären Substandardmerkmalen. Ihre Existenz verdanken diese Merkmale der oben beschriebenen Normskepsis seit der Mitte des 20. Jahrhunderts (Bellmann 1983). Der regionale Standard hat meiner Ansicht nach vor allem diese beiden Quellen der Abweichung von der Norm: die primären Substandardmerkmale, die ihren Ursprung in den zugrunde liegenden Dialekten einer Region haben, und die sekundären Substandardmerkmale. ${ }^{11}$

Folgende Tabelle (Tabelle 2) gibt einen Überblick über einige von mir untersuchte sekundäre Substandardmerkmale (oder: Allegromerkmale, wie ich sie an anderer Stelle genannt habe, Spiekermann 2005 u. a.). Alle Merkmale sind überregional verbreitet, d. h. sie kommen nicht nur in den Daten aus Baden-Württemberg, die ich untersucht habe, vor, sondern im Prinzip in allen deutschsprachigen Regionen.

\footnotetext{
${ }^{11}$ Lenz (2003) zählt daneben auch Hyperformen zu Merkmalen standardnaher Substandardvarietäten. Nach meinen Untersuchungen ist das - zumindest heute in Baden-Württemberg - fast nicht mehr der Fall.
} 


\begin{tabular}{|l|l|l|}
\hline Nr. & Merkmal & Beispiel \\
\hline 1 & Tilgung von Schwa in der 1. Ps. Sg. & (ich) $<$ geh $>$ statt $<$ gehe $>$ \\
\hline 2 & Klitisierung von $<$ es $>$ & $($ er $)<$ hat's $>$ statt $<$ hat es $>$ \\
\hline 3 & Tilgung von $/$ t/ in $<$ ist $>$ & $<$ is $>$ statt $<$ ist $>$ \\
\hline 4 & Tilgung von $/$ t/ in $<$ nicht $>$ & $<$ nich $>$ statt $<$ nicht $>$ \\
\hline 5 & Reduzierung unbestimmter Artikel & $<$ n, ne $>$ statt $<$ ein, eine $>$ \\
\hline
\end{tabular}

Tabelle 2: Übersicht sekundäre Substandardmerkmale

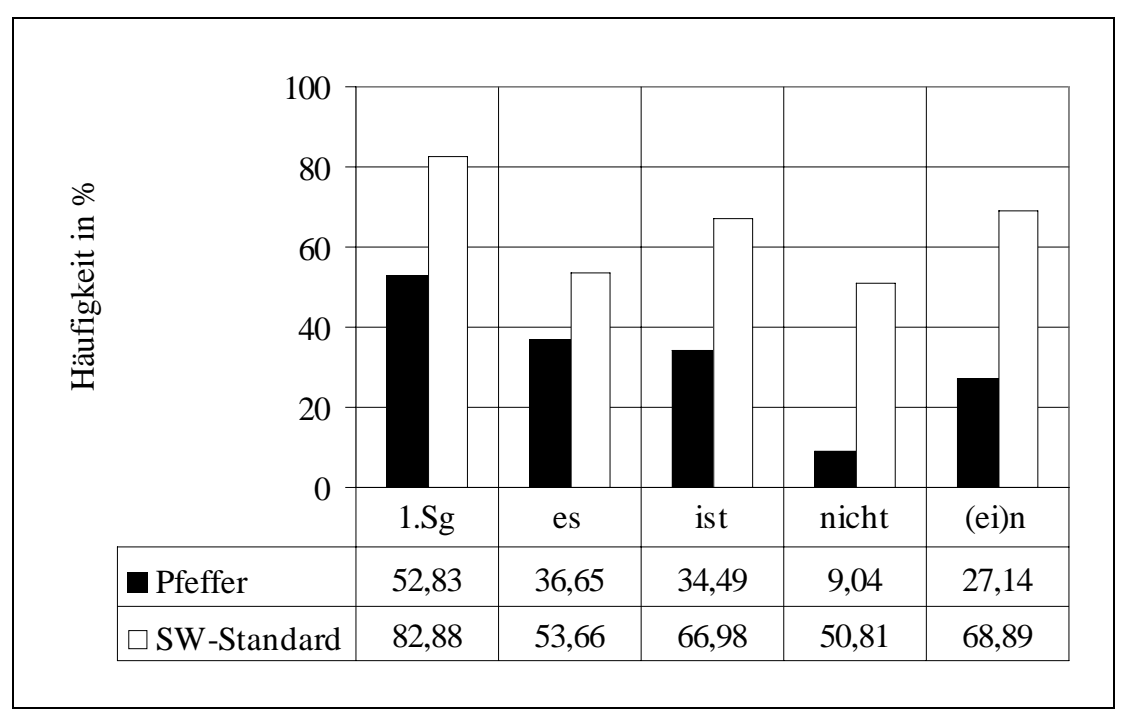

Diagramm 2: Realisierungshäufigkeiten sekundäre Substandardmerkmale

Diachron nimmt die Zahl der normabweichenden Realisierung deutlich zu. Diagramm 2 zeigt, dass in meinen Daten aus den Jahren 2001 bis 2003 sekundäre Substandardmerkmale in über $50 \%$ der Fälle realisiert sind, die Tilgung eines auslautenden Schwas in der ersten Person Singular der Verben sogar in über $80 \%$. Dies deckt sich auch mit den Ergebnisse der Studie von Berend (s. o.), die ganz ähnliche Merkmale untersucht hat und zu vergleichbaren Ergebnissen gekommen ist: Der Normstandard wird an dieser Stelle deutlich zurückgedrängt.

Dass wir es hier mit einer dynamischen Entwicklung zu tun haben, kann man auch daran erkennen, dass sich unterschiedliche Altersgruppen in Bezug auf die Verwendung sekundärer Substandardmerkmale unterschiedlich verhalten. Besonders häufig sind sie bei Vertretern und Vertreterinnen der jungen Generation anzutreffen. Versucht man alleine auf der Basis der gesprochenen Sprache eine genaue regionale Einordnung der Sprecherinnen vorzunehmen, so wird dies nicht ohne weiteres möglich sein. Sprecher und Sprecherinnen der jüngeren Generation (und einer bestimmten höheren Bildungsschicht) gebrauchen eine Standardvarietät, die praktisch keine Dialektmerkmale mehr enthält. Dies bedeutet allerdings nicht zwangsläufig, dass sie nicht in der Lage wären, Dialekt und Regionalsprache zu sprechen. Tatsächlich ist es so, dass sie - selbst wenn sie noch Dialekt sprechen - durchaus in der Lage sind, einen nahezu dialektfreien Standard zu artikulieren. Ihre Standardvarietät enthält jedoch andere Merkmale, die von der Explizitlautung einer Standardsprache abweichen, und zwar in sehr großer Zahl, nämlich die oben bereits beschriebenen "umgangssprachlichen" Merkmale (oder: sekundäre Substandardmerkmale). Diese werden in Häufigkeiten nahe der $100 \%$ realisiert.

Die von mir vorgestellten Beispiele zeigen - wie ich denke - relativ eindeutig, dass es sich bei der Standardsprache nicht um die variationsfreie, einheitliche Sprachform handelt, als die 
sie manchmal erscheint. Wir haben es vielmehr mit einer Gruppe von Varietäten zu tun, die sich in primären und sekundären Substandardmerkmalen z. B. deutlich vom Normstandard unterscheiden. Diese Unterschiede sind aber nicht beliebig, sondern dergestalt, dass sie systematisch beschrieben und damit grundsätzlich auch z. B. für den fremdsprachlichen Deutschunterricht nutzbar gemacht werden können.

\section{Variation und der Unterricht "Deutsch als Fremdsprache"}

Im Unterricht "Deutsch als Fremdsprache" wird in der Regel das "standardsprachliche Deutsch" (Hernig 2005: 31) vermittelt. Hierfür gibt es eine Reihe guter Gründe:

- Die Standardsprache ist normiert, d. h. bei ihrer Vermittlung kann auf unterschiedliche Kodizes als Referenzrahmen zurückgegriffen werden.

- Sie verfügt über ein hohes Prestige.

- Die Standardsprache weist einen großen Kommunikationsradius auf.

In Überblickswerken zum Unterricht "Deutsch als Fremdsprache" (Huneke/Steinig 1997, Hernig 2005) wird mit Bezug auf die Frage des Kommunikationsradius auf die Variabilität des Deutschen insbesondere mit dem Hinweis auf das Konzept der Plurizentrizität aufmerksam gemacht. Der Hinweis darauf, dass Nicht-Muttersprachler des Deutschen, wenn sie ausschließlich den (variationsfreien) Normstandard erlernen, sich durchaus Muttersprachlern in unterschiedlichen Regionen gegenüber verständlich machen können (so Hernig 2005), berücksichtigt alleine den Aspekt der Sprachproduktion, nicht aber den der Sprachrezeption. Was geschieht, wenn Deutschlerner mit sehr guten Kenntnissen in der variationsfreien Standardsprache auf Muttersprachlern treffen, die sich selbst nur in ihren regionalen Varietäten ausdrücken können? Selbst der Gebrauch regionaler Standardvarietäten kann zu Kommunikationsproblemen führen, auf die der Deutschunterricht vorbereiten kann.

Dass es im Gebrauch und in der Kenntnis um regional eingeschränkt gebräuchliche Formen im Standard Unterschiede zwischen Muttersprachlern und Nicht-Muttersprachlern des Deutschen gibt, hat eine Untersuchung zu syntaktischen Erscheinungen gezeigt, die von Studierenden an der Universität Freiburg angefertigt wurde (Cho/Gechter 2007). In Form einer Fragebogenuntersuchung wurden 26 Nicht-Muttersprachler und 29 Muttersprachler des Deutschen (Studierende an der Universität Freiburg) bzgl. ihres subjektiven Wissens über und des persönlichen Gebrauchs von bestimmten syntaktischen Phänomen befragt, die nach Maßgabe der Forschungsliteratur als Merkmale von Regionalsprachen und regionalen Standardvarietäten bzw. als sekundäre Substandardmerkmale (im Sinne Albrechts 2005) betrachtet werden können. Folgende Tabelle (Tabelle 3) gibt einen Überblick über einige der untersuchten Merkmale (die Merkmale 1 und 2 sind Beispiele für sekundäre, die Merkmale 3 und 4 für primäre Substandardmerkmale): 


\begin{tabular}{|c|l|l|}
\hline Nr. & Merkmal & Beispiel \\
\hline 1 & Hauptsatzstellung im <weil>-Nebensatz & $\begin{array}{l}\text { Ich konnte gestern nicht in die Stadt kommen, } \\
\text { (a) weil es geregnet hat. (Norm) } \\
\text { (b) weil es hat geregnet. (gesprochener Substandard) }\end{array}$ \\
\hline 2 & <tun>-Fügung & $\begin{array}{l}\text { (a) Das überlegen wir uns später. (Norm) } \\
\text { (b) Das tun wir uns später überlegen. (gesprochener } \\
\text { Substandard) }\end{array}$ \\
\hline 3 & <am>-Progressiv (Rheinische Verlaufs-form) & $\begin{array}{l}\text { (a) Ich schreibe gerade einen Brief. (Norm) } \\
\text { (b) Ich bin gerade einen Brief am schreiben. } \\
\text { (ursprünglich westmitteldt./rheinisch) }\end{array}$ \\
\hline 4 & Gebrauch der Präpositionen <um> oder <für> $>\begin{array}{l}\text { Ich habe kein Geld, } \\
\text { (a) um ein neues Auto zu kaufen. (Norm) } \\
\text { (b) für ein neues Auto zu kaufen. (u. a. mitteldt.) }\end{array}$ \\
\hline
\end{tabular}

Tabelle 3: Übersicht über untersuchte syntaktische Substandardformen in Cho/Gechter 2007, Auswahl

Die Probanden, die sich an der Fragebogenerhebung beteiligten, wurden gebeten, entlang einer Skala anzugeben, wie häufig sie die vorgegebenen Alternativen verwendeten bzw. ob ihnen die Formen überhaupt bekannt waren. Neben der normsprachlichen und der erwarteten substandardsprachlichen Realisierung wurden weitere Alternativen (auch völlig absurde) angegeben, die dazu verwendet wurden, die Zuverlässigkeit der gemachten Aussagen zu überprüfen. Jede der angebotenen Alternativen wurde von den Probanden bewertet. Auch wenn Cho/Gechter (2006: 38) feststellen, dass sich Muttersprachler und NichtMuttersprachler nach den Ergebnissen ihrer Untersuchung in ihrem Sprachgebrauch nicht wesentlich unterscheiden, lassen sich doch einige interessante Tendenzen festhalten, die einen Einblick in das Wissen über und in die Verwendungsgewohnheiten regionaler Standardvarietäten erlauben: Zum einen ist die Tendenz bei der überwiegenden Mehrheit der untersuchten syntaktischen Konstruktionen erkennbar, dass Nicht-Muttersprachler Substandardformen (sowohl primäre als auch sekundäre) weniger häufig verwenden als Muttersprachler des Deutschen. Zum anderen (und dies ist der bedeutsamere Punkt) stellte sich heraus, dass Nicht-Muttersprachler viele der untersuchten Merkmale im Gegensatz zu den Muttersprachlern häufig nicht kannten. ${ }^{12}$ Cho/Gechter (2006: 38f.) erklären diesen wenig überraschenden Befund damit, dass der Unterricht Deutsch als Fremdsprache, den viele der Nicht-Muttersprachler in ihren Heimatländern genossen haben, ausschließlich auf grammatisch korrekte, den Normen entsprechende Formen ausgerichtet gewesen ist und viele der Probanden auch jetzt noch in ihrem aktiven Sprachgebrauch diese Formen bevorzugen. Ein Unterricht, der die regionale/nationale Variation in der Standardsprache nicht berücksichtigt, muss zwangsläufig dazu führen, dass Nicht-Muttersprachler des Deutschen eine andere Standardsprache sprechen als Muttersprachler (was ja nicht Ziel des DaFUnterrichts sein kann) und viele Bestandteile der Sprache - seien es grammatischen Konstruktionen oder lexikalische Einheiten -, die für Muttersprachler vollkommen alltäglich und normal sind, nicht erfassen können.

\subsection{Berücksichtigung unterschiedliche Standardvarietäten}

Was den tatsächlichen Unterricht und die Berücksichtigung nationaler und regionaler Standardvarietäten dort anbetrifft, wird - soweit ich es überblicken kann - ein deutlicher

\footnotetext{
12 Es ist hierbei allerdings anzumerken, dass die Ergebnisse sehr heterogen sind. Besonders innerhalb der Gruppe der Nicht-Muttersprachler tauchen durchaus unterschiedliche Ergebnisse auf, die damit zu tun haben, wie groß die Deutschkenntnisse sind und nicht zuletzt auch wie lange die Probanden sich in einer deutschsprachigen Umgebung aufhalten. Je länger dies der Fall ist, desto größer sind erwartungsgemäß die Kenntnisse über die Substandardformen.
} 
Unterschied zwischen den beiden Varietäten bzw. Varietätengruppen gemacht. Die Berücksichtigung nationaler Standardvarietäten ist von unterschiedlichen Seiten in den letzten Jahren wiederholt angemahnt worden (König 1997, Takahashi 1999, Hensel 2000, Baßler/Spiekermann 2001 und zuletzt Hägi 2005). Sara Hägi hat in ihrer Dissertation aus dem Jahr 2005 sehr ausführlich dargestellt, wie nationale Varietäten des Deutschen bereits heute in DaF-Lehrwerken berücksichtigt werden. Insgesamt widmen sich die Lehrwerke den Varietäten nur sehr zaghaft, man kann aber immerhin feststellen, dass in sprachlichen und landeskundlichen Übungen besonders lexikalische nationale Varianten diskutiert und vorgestellt werden. Regionale Standardvarietäten werden in Form von relativ authentischen Tonbeispielen ebenfalls behandelt (cf. Baßler/Spiekermann 2001/02), ihre Thematisierung erfolgt jedoch relativ unsystematisch. Dies liegt u. a. daran, dass die linguistische Forschung in diesem Bereich noch nicht so weit fortgeschritten ist, dass eine systematische Aufbereitung dieser Varietäten bisher möglich gewesen wäre (cf. auch die Rezension zum Sammelband "Regionale Standardvarietäten" von Harald Baßler 2004). Neuere Arbeiten in diesem Bereich (Lenz 2003, Berend 2005, Spiekermann 2006b u. a.) beginnen diese Lücke aber zu schließen. Fehlende Forschungsarbeiten zu nationalen Standardvarietäten kann man als Grund für die mangelhafte Berücksichtigung dieser Varietäten aber kaum gelten lassen. Die Arbeiten von Ammon, Clyne u. a. haben ein relativ komplexes und umfassendes Bild entstehen lassen, das als Grundlage für die Berücksichtigung nationaler Varietäten herangezogen werden kann. Es gibt aus dieser Perspektive keinen Grund, nicht stärker auf nationale und regionale Standardvarietäten einzugehen.

Als Ursachen für die relativ zurückhaltende Berücksichtigung standardsprachlicher Variation im DaF-Unterricht nennt Martin Durell (2006: 118) in erster Linie die "Einstellung zur Variation im deutschen Sprachgebiet" und die "ideologisch bedingte [...] Vorstellung einer variationslosen Standardsprache." Er vertritt die Meinung, dass zur Erstellung von Unterrichtsmaterialien keine präskriptiven Kodizes notwendig sind. Deskriptive Darstellungen von Unterschieden zwischen regionalen Varietäten können für die Erstellung von Unterrichtsmaterialien ebenfalls herangezogen werden. Dieser Meinung schließe ich mich an. Neuere linguistische Forschungsarbeiten bieten durchaus die Möglichkeit, sich differenziert mit standardsprachlicher Variation zu befassen und diese $\mathrm{zu}$ beschreiben. In welchem Umfang dies geschehen soll bzw. kann, lässt sich pauschal nicht entscheiden. Es gibt jedoch sicherlich einige grundsätzliche Punkte, die in diesem Zusammenhang beachtet werden sollten.

\subsection{Möglichkeiten der Berücksichtigung}

Meiner Ansicht nach muss die Frage nach den Möglichkeiten der Berücksichtigung unterschiedlicher Standardvarietäten im Unterricht "Deutsch als Fremdsprache" aus unterschiedlichen Perspektiven beantwortet werden:

- Was ist das Ziel des Unterrichts?

- Was sind die Ziele der DaF-Lerner/Lernerinnen?

- Wie ist der Lernort zu berücksichtigen?

- Wie lassen sich individuelle Ziele einbeziehen?

Oben wurde als Ziel des Deutschunterrichts die Vermittlung des standardsprachlichen Deutsch wenn auch nicht explizit aber doch indirekt eingeführt. Unterrichtsgegenstand ist die Standardsprache. Dieser Standpunkt ist zum einen ungenau (da es unterschiedliche Standardvarietäten gibt), er ist außerdem nicht unwidersprochen. Es gibt Positionen, nach denen die Alltagssprache Ziel des Unterrichts sein sollte (Günthner 2002, Durrell 2006). Schließlich geht es um den Aufbau kommunikativer Fähigkeiten, so dass die Inhalte des Deutschunterrichts sich an der Alltagssprache orientieren müssen. Alltagssprache ist nun aber 
durchaus nicht mit einer variationsfreien Standardsprache gleichzusetzen. Ist also das Ziel des Unterrichts die Vermittlung der Alltagssprache, muss sich der Unterricht auch intensiv mit Varietäten neben dem variationsfreien Standard befassen. Nationale und regionale Standardvarietäten bieten hier ausreichend Material und stellen eine sinnvolle Alternative zur bloßen Vermittlung standardsprachlicher Normen.

Aus Befragungen ist bekannt, dass Lerner und Lernerinnen oft offener für die Behandlung regionaler Varietäten sind als ihre Lehrer und Lehrerinnen (Baßler/Spiekermann 2001, auch Davies 2006). Gerade was passive Kenntnisse in regionalen Varietäten anbetrifft, wünscht sich zumindest ein Teil der Lerner/Lernerinnen ein intensivere Auseinadersetzung als Lehrer/Lehrerinnen dies oft für nötig und vor allem für möglich halten (cf. Baßler/Spiekermann 2001), wobei individuelle Unterschiede in den Lernzielen der Lernenden bestehen, die im Unterricht vermutlich kaum erschöpfend berücksichtigt werden können. ${ }^{13}$ Tatsächlich wird von Seiten der Lehrenden oft argumentiert, dass die knappe zur Verfügung stehende Zeit im Unterricht dazu genutzt werden muss, die standardsprachliche Norm zu vermitteln. Ohne Zweifel sind die Möglichkeiten zur Erweiterung der Inhalte im Unterricht begrenzt. Die Vermittlung einheitlicher grammatischer Regeln und eines einheitlichen Grundwortschatzes, wie die genormte und kodifizierte Standardsprache sie vorgibt, sollte meiner Ansicht nach auch weiterhin das Zentrum des DaF-Unterrichts ausmachen. Man könnte jedoch bei bereits bestehenden und in den Unterricht integrierten Einheiten zur Landeskunde oder zu sprachlicher Variation, wie sie z. B. in Baßler/Spiekermann (2001/02) oder Hägi (2006) für einige Lehrwerke beschrieben sind, eine Schwerpunktverschiebung vornehmen bzw. eine inhaltliche Fokussierung erreichen. Wesentlich scheint mir in diesem Zusammenhang die Frage des Lernortes. Es ist sicherlich sehr sinnvoll, ortsübliche regionale Varietäten zu thematisieren, wenn der DaF-Unterricht in einem deutschsprachigen Land stattfindet. Die Kenntnisse in der Alltagssprache lassen sich durch authentische Sprachbeispiele, wie sie immer wieder gefordert werden, gezielt verbessern. Man sollte den Lernortvorteil, den der DaF-Unterricht vor Ort mit sich bringt, zu nutzen versuchen, auch indem man nationale und regionale Standardvarietäten der Regionen um den Unterrichtsort stärker in den Fokus rückt. Hierzu ist allerdings nicht allein die Bereitschaft der Lernenden und Lehrenden, sondern auch geeignetes Unterrichtsmaterial notwendig, an dem es zur Zeit noch weitgehend fehlt. Besonders an diesem Punkt kann noch eine Menge geleistet werden.

\section{$4 \quad$ Moderne soziolinguistische Forschung und der Unterricht "Deutsch als Fremdsprache"}

Die deutsche Standardsprache ist in ihrer geschriebenen und besonders in ihrer gesprochenen Form keine einheitliche Sprache. Es sind nationale und regionale Standardvarietäten, mit denen die Lerner von Deutsch als Fremdsprache - besonders wenn sie sich in deutschsprachigen Ländern bewegen - in ihrem Alltag und über moderne Massenmedien konfrontiert werden, und nicht der genormte Standard, der häufig noch der alleinige Gegenstand des DaF-Unterrichts ist. Standardsprachliche Variation ist in den vergangenen Jahren in soziolinguistischen Arbeit intensiv untersucht worden und wird - soweit man es heute sagen kann - die soziolinguistische Forschung auch der kommenden Jahre als eines ihren zentralen Themen bestimmen. Die Forschungslage wird sich mit anderen Worten immer weiter verbessern. Damit wird auch die Voraussetzung für eine intensive Beschäftigung mit

\footnotetext{
13 Die Gründe für das Erlernen der deutschen Sprache sind individuell natürlich sehr unterschiedlich und reichen von einem reinen Interesse an der deutschen Sprache und Kultur bis hin zu rein beruflichen Qualifikationsmaßnahmen. Die Frage, ob die deutsche Sprache später nur in schriftlicher oder auch in mündlicher Kommunikation verwendet werden soll, beeinflusst die Bereitschaft, sich über die genormte Standardsprache hinaus mit sprachlicher Variation auseinandersetzen.
} 
unterschiedlichen standardsprachlichen Varietäten im Unterricht "Deutsch als Fremdsprache" geschaffen.

\section{Literaturangaben}

Albrecht, Jörn (2005): "Die Gemeinsprache als jüngstes Stadium der Nationalsprache". In: Lenz, Alexandra/Mattheier, Klaus J. (eds.): Varietäten - Theorie und Empirie. Frankfurt/Main etc.: 127-140.

Ammon, Ulrich (1995): Die deutsche Sprache in Deutschland, Österreich und der Schweiz. Das Problem der nationalen Varietäten. Berlin, New York.

Ammon, Ulrich (1998): "Plurinationalität oder Pluriarealität? Begriffliche und terminologische Präzisierungsvorschläge zur Plurizentrizität des Deutschen - mit einem Ausblick auf ein Wörterbuchprojekt". In: Ernst, Peter/Patocka, Franz (eds.): Deutsche Sprache in Raum und Zeit. Festschrift für Peter Wiesinger zum 60. Geburtstag. Wien: 313-322.

Ammon, Ulrich (2003): "Dialektschwund, Dialekt-Standard-Kontinuum, Diglossie. Drei Typen des Verhältnisses Dialekt - Standardvarietät im deutschen Sprachgebiet". In: Androutsopoulos, Jannis K./Ziegler, Evelyn (eds.): 'Standardfragen'. Soziolinguistische Perspektiven auf Sprachgeschichte, Sprachkontakt und Sprachvariation. Frankfurt/Main etc.: $8-17$.

Ammon et al. (2004): Variantenwörterbuch des Deutschen. Die Standardsprache in Österreich, der Schweiz und Deutschland sowie in Liechtenstein, Luxemburg, Ostbelgien und Südtirol. Berlin/New York.

Auer, Peter (2004): "Sprache, Grenze, Raum". Zeitschrift für Sprachwissenschaft 23 (2): 149_ 179.

Auer, Peter (2005): "Europe's Sociolinguistic Unity, or A Typology of European Dialect/Standard Constellations". In: Delbecque, Nicole/Auwera, Johan van der/Geeraerts, Dirk (eds.): Perspectives on Variation. Berlin/New York: 7-42.

Barbour, Stephen/Stevenson, Patrick (1998): Variation im Deutschen. Soziolinguistische Perspektiven. Berlin/New York.

Baßler, Harald/Spiekermann, Helmut (2001): "Dialekt und Standardsprache im DaFUnterricht. Wie Lehrer urteilen - wie Schüler urteilen". Linguistik online 9, 2/01. http://www.linguistik-online.com/9_01/BasslerSpiekermann.html.

Baßler, Harald/Spiekermann, Helmut (2001/02): "RegionaleVarietäten des Deutschen im Unterricht 'Deutsch als Fremdsprache'". Deutsch als Fremdsprache 38 (4): 205-213; 39 (1): 31-35.

Baßler, Harald (2004): "Rezension zu Elisabeth Knipf-Konlósi/Nina Berend (eds.): Regionale Standards. Sprachvariation in den deutschsprachigen Ländern". Deutsch als Fremdsprache 41 (1): 54-56.

Bellmann, Günter (1983): "Probleme des Substandards im Deutschen". In: Mattheier, Klaus Jürgen (ed.): Aspekte der Dialekttheorie. Tübingen: 105-130.

Berend, Nina (2005): "Regionale Gebrauchsstandards. Gibt es sie und wie kann man sie beschreiben?" In: Eichinger, Ludwig M./Kallmeyer, Werner (eds.): Standardvariation. Wie viel Variation verträgt die deutsche Sprache? Berlin/New York: 143-170.

Bichel, Ulf (1973): Problem und Begriff der Umgangssprache in der germanistischen Forschung. Tübingen.

Cho, Yun-Jung/Gechter, Dorothea (2007): Eine Umfrage zur deutschen Umgangssprache. Hauptseminarsarbeit. Universität Freiburg, Deutsches Seminar I.

Christen, Helen (2001): "Die regionalen Besonderheiten der deutschen Standardsprache in der Schweiz". In: Berend, Nina/Knipf-Komlósi, Elisabeth (eds.): Regionale Standards. Budapest/Pécs: 120-159. 
Clyne, Michael (1992): "German as a Pluricentric Language". In: Clyne, Michael (ed.): Pluricentric Languages. Differing Norms in Different Nations. Berlin/New York: 117-147.

Davies, Winifred (2006): "Normbewusstsein, Normkenntnis und Normtoleranz von Deutschlehrkräften". In: Neuland, Eva (ed.): Variation im heutigen Deutsch. Perspektiven für den Sprachunterricht. Frankfurt/Main etc.: 483-491.

Dittmar, Norbert (1997): Grundlagen der Soziolinguistik. Tübingen.

Durrell, Martin (2006): "Deutsche Standardsprache und Registervielfalt im Daf-Unterricht". In: Neuland, Eva (ed.): Variation im heutigen Deutsch. Perspektiven für den Sprachunterricht. Frankfurt/Main etc.: 111-122.

Eichhoff, Jürgen (1977): Wortatlas der deutschen Umgangssprache. Erster Band. Bern/München.

Eichinger, Ludwig M. (2001): "Sprache und Sprachgebrauch im Süden Deutschlands". In: Berend, Nina/Knipf-Komlósi, Elisabeth (eds.): Regionale Standards. Budapest/Pécs: 61-94.

Elspaß, Stefan/Möller, Robert (2006): "Internet-Exploration. Zu den Chancen, die eine Online-Erhebung regional gefärbter Alltagssprache bietet". Osnabrücker Beiträge zur Sprachtheorie 71: 101-118.

Flüe-Fleck, Hanspeter/Hove, Ingrid (1994): "Schweizerhochdeutsch. Ein Thema im DaFUnterricht?" In: Breitung, Horst (ed.): Phonetik, Intonation, Kommunikation. München: 118.

Günthner, Susanne (2002): "Konnektoren im gesprochenen Deutsch. Normverstoß oder funktionale Differenzierung?" Deutsch als Fremdsprache 9/2002: 67-74.

Hägi, Sara (2005): Nationale Varietäten im Unterricht Deutsch als Fremdsprache. Frankfurt/Main etc.

Hensel, Sonja (2000): "Welches Deutsch sollen wir lehren?" Zielsprache Deutsch 31 (1), 3139.

Hernig, Marcus (2005): Deutsch als Fremdsprache. Eine Einführung. Wiesbaden.

Hove, Ingrid (1995): "Zur Aussprache der Standardsprache in der deutschen Schweiz". In: Löffler, Heinrich (ed.): Alemannische Dialektforschung. Bilanz und Perspektiven. Beiträge zur 11. Arbeitstagung Alemannischer Dialektologen. Tübingen etc.: 291-293.

König, Werner (1997): "Phonetisch-phonologische Regionalismen in der deutschen Standardsprache. Konsequenzen für den Unterricht 'Deutsch als Fremdsprache'". In: Stickel, Gerhard (ed.): Varietäten des Deutschen. Regional- und Umgangssprachen. Berlin/New York: 246-270.

Krumm, Hans-Jürgen (2006): "Normen, Varietäten und Fehler. Welches Deutsch soll der Deutsche als Fremdsprache-Unterricht lehren?" In: Neuland, Eva (ed.): Variation im heutigen Deutsch. Perspektiven für den Sprachunterricht. Frankfurt/Main etc.: 459-468.

Lenz, Alexandra N. (2003): Struktur und Dynamik des Substandards. Eine Studie zum Westmitteldeutschen (Wittlich/Eifel). Stuttgart.

Löffler, Heinrich ( $\left.{ }^{2} 1994\right)$ : Germanistische Soziolinguistik. Berlin.

Mattheier, Klaus J. (2003): "German". In: Deumert, Ana/Vandenbusche, Wim (eds.): Germanic standardizations. Past to present. Philadelphia: 211-244.

Niebaum, Hermann/Macha, Jürgen (2006): Einführung in die Dialektologie des Deutschen. 2., neubearb. Aufl. Tübingen.

Schmidt, Jürgen Erich (1998): "Moderne Dialektologie und regionale Sprachgeschichte." Zeitschrift für deutsche Philologie 117: 163-179.

Spiekermann, Helmut (2005): "Regionale Standardisierung, nationale Destandardisierung". In: Eichinger, Ludwig M./Kallmeyer, Werner (eds.): Standardvariation. Wie viel Variation verträgt die deutsche Sprache? Berlin/New York: 100-125.

Spiekermann, Helmut (2006): "Standardsprache als regionale Varietät. Regionale Standardvarietäten". Osnabrücker Beiträge zur Sprachtheorie 71: 81-99. 

nationale Standardvarietäten - regionale Standardvarietäten

Spiekermann, Helmut (2006 $)$ : Standardsprache in Baden-Württemberg. Eine empirische Untersuchung zu konstitutiven Merkmalen regionaler Standardvarietäten. Habilitationsschrift, Albert-Ludwigs-Universität Freiburg.

Takahashi, Hideaki (1999): "Verschiedene Varietäten des Deutschen und deren Beziehung zum Unterricht Deutsch als Fremdsprache". Zeitschrift für Angewandte Linguistik 31: 109123. 